\title{
CARACTERISTICAS BROMATOLOGICAS DE DIECISEIS ESPECIES HIDROBIOLOGICAS DE LA AMAZONIA PERUANA EN EPOCA DE CRECIENTE
}

Juan Pedro Cortez Solís ( ${ }^{*}$ )

\section{RESUMEN}

El presente trabajo, tiene por finalidad, conocer los valores proteicos y las características porcentuales de sus otros componentes, como son: humedad, grasas, carbohidratos y cenizas, de 16 especies hidrobiológicas seleccionadas en época de creciente.

Los resultados indican que las especies de mayor contenido proteico son el "Paiche' Arapaima gigas con $20.6 \%$, la 'corvina' Plagiosción squamosissimus con $20.18 \%$ y la" arahuana" O steoglossum bicirrhosum con $20.10 \%$; en contenido de grasa los de mayor porcentaje son "maparate" Hypopthalmus edentatus con $15.80 \%$, "palometa" M ylossoma duriventris con 13.32\%; en contenido de sales minerales los de mayor contenido son "gamitana" Colossoma macropomum con $3.41 \%$ y el "maparate" con $2.30 \%$; en cuanto a humedad las especies de mayor contenido son La "ractacara" Curimata vittata con $80.41 \%$ y "carachama"Pterygoplighthys multiradiatus con $80.02 \%$. También las especies han quedado clasificadas según su contenido graso en: magras (carachama, corvina, paiche y arahuana), semigrasos (lisa, sábalo, sardina, y ahuarachi, ractacara, yulilla y dorado) y grasas (paco, gamitana, palometa, boquichico y maparate). El presente será una importante contribución para el conocimiento de las bondades naturales de los peces amazónicos y también para estudios tecnológicos tendientes a su aprovechamiento en diferentes formas de conservación.

Palabras Claves: A nálisis Bromatológico, Humedad, Proteina, Crasas, Carbohidratos, Cenizas, Peces A mazónicos.

\section{INTRODUCCION}

La A mazonía Peruana, con su compleja red hídrica de ríos, quebradas y lagunas, alberga una gran variedad de recursos hidrobiológicos (peces, moluscos y crustáceos, etc. ), la mayor parte de los cuales son considerados aptos para el consumo humano.

INSTITUTO DE INVESTIGACIONES DE LA AMAZONIA PERUANA - IIAP Av. A belardo Quiñónez Km. 2.5 A partado 784 Iquitos - Perú 
EI IIAP, consciente de la importancia que tiene la debida utilización de estos recursos en la alimentación de la población amazónica, ha centrado sus esfuerzos en estudiarlos desde el punto de vista tecnológico para su adecuada utilización en diversos productos de consumo humano. Para ello se han realizado análisis bromatológicos de las principales especies de consumo que indican que los recursos amazónicos con mínimas diferencias se encuentran en los mismos rangos nutricionales de las especies marinas. Teniendo a su favor un mayor contenido de sales minerales.

\section{MATERIALES Y METODOS}

Para los efectos del estudio se seleccionaron 16 especies hidrobiológicas, de acuerdo a su importancia comercial y disponibilidad, las que se aprecian en la Tabla 1. Estas especies fueron adquiridas al estado fresco en los mercados y puertos de Iquitos,

Los análisis bromatológicos se realizaron por triplicado, en forma mensual, abarcando desde el mes de febrero a abril de 1987, para su realización se utilizaron los laboratorios de Industrias Químicas de la UNAP y del IIAP.

Los análisis físicos comprendieron el estado de frescura, longitud, peso, humedad, etc; los análisis químicos la determinación de proteínas, grasas, carbohidratos y cenizas, utilizándose para estos análisis la parte comestible homogenizada del pescado.

Los métodos de análisis utilizados fueron los propuestos para productos pesqueros por el Ministerio de Salud y por los Institutos Nacionales de Salud (Composición de A limentos Peruanos, 1975); el método se señala en la Tabla 2.

\section{RESULTADOS}

\subsection{Aspectos de las muestras}

L as especies presentaron buen y regular estado de frescura (pescado refrigerado), la longitud, peso y altura promedios, así como su procedencia, se señalan en la Tabla 3.

La ractacara y palometa fueron los más pequeños en promedio, con 14.6 y $13.4 \mathrm{~cm}$. respectivamente, y los de mayor tamaño promedio fueron el paiche y dorado, con $1.74 \mathrm{~m}$. y $75.2 \mathrm{~cm}$. Respectivamente; en peso, estas mismas especies tienen la mayor o menor clasificación. La mayoría de las especies fueron capturadas en el río A mazonas (áreas cercanas a Iquitos) y, en segundo plano, se tiene a los ríos U cayali y M arañón, con pescado refrigerado.

\subsection{Aspectos broniatológicos de las muestras}

Los resultados de los análisis bromatológicos se muestran en la Tabla 3 y las características principales de sus parámetros son las sgtes.: 


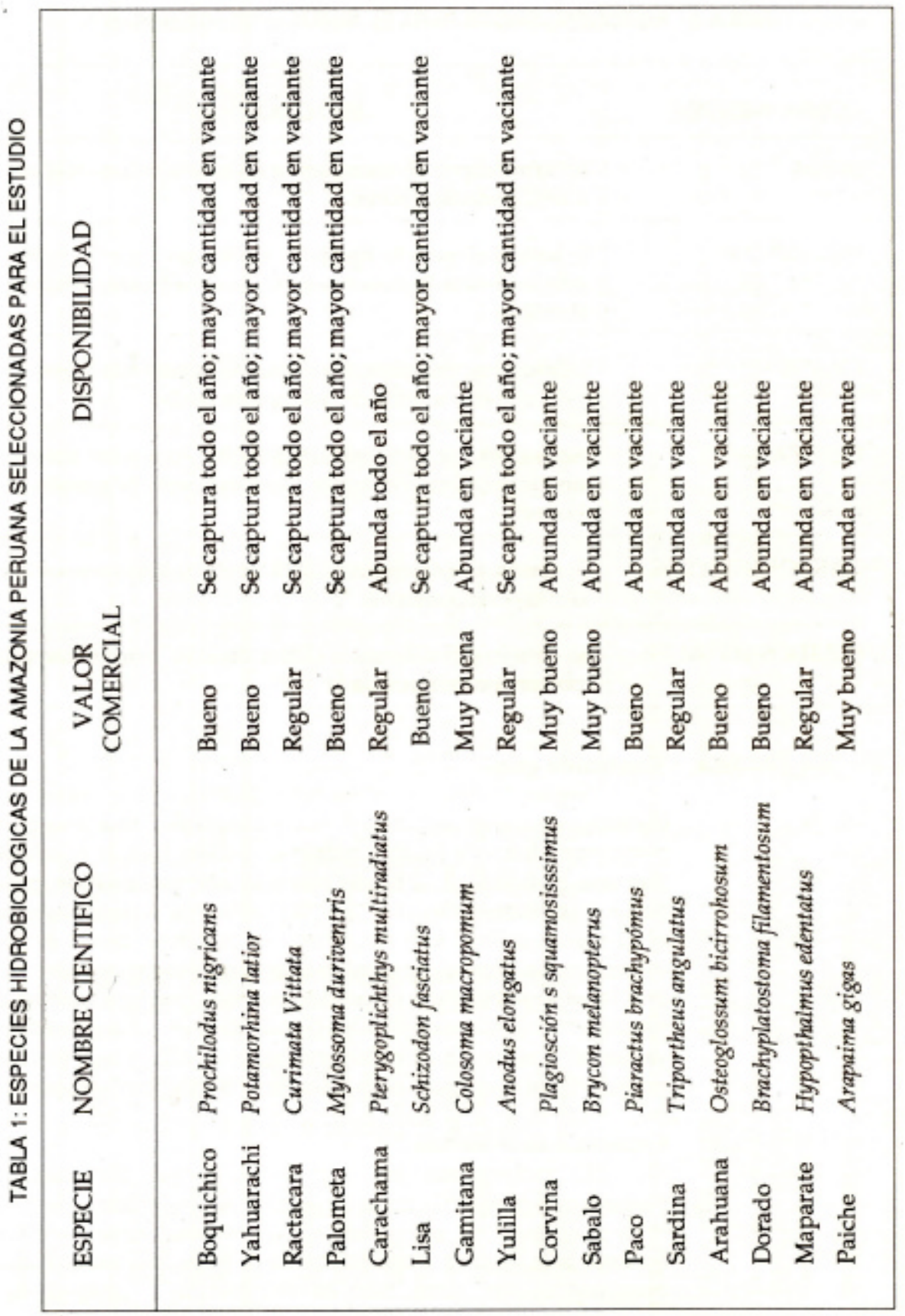


TABLA 2 : METODOS USADOS PARA EL ANALISIS DE MUESTRAS

\begin{tabular}{|l|l|}
\hline COMPONENTES & \multicolumn{1}{|c|}{ METODOS } \\
\hline AGUA & $\begin{array}{l}\text { Se terminó por desecación de las muestras en estufas a } \\
110^{\circ} \mathrm{C} \text { durante } 6 \text { horas. }\end{array}$ \\
\hline PROTEINAS & $\begin{array}{l}\text { Se utilizó el método Kjeldahl, modificado por Gunning, } \\
\text { con la adición degránulos de alúmina selenizas y sulfato } \\
\text { de cobre. }\end{array}$ \\
\hline GRASA & $\begin{array}{l}\text { La muestra, previamente desecada,se sometió a extrac- } \\
\text { ción con éter anhidrido, en equipo Soxlet. }\end{array}$ \\
\hline CENIZAS & $\begin{array}{l}\text { Se realizó por incineración a } 600^{\circ} \text { C durante el tiempo } \\
\text { necesario para conseguir cenizas libres de carbón (6 } \\
\text { horas) }\end{array}$ \\
\hline CARBOHIDRATOS & $\begin{array}{l}\text { Se obtuvo por diferencia (100\%) de la muestra menos los } \\
\text { demás componentes) }\end{array}$ \\
\hline SOLIDOS TOTALES & $\begin{array}{l}\text { Se obtuvo por diferencia (100\% de la muestra menos el } \\
\text { porcentaje de humedad) }\end{array}$ \\
\hline
\end{tabular}

\subsubsection{Contenido graso}

Según Vallejos y M enchola, (1984), a las grasas les corresponde una importancia destacada como fuente de energía y como portadores de ácido y grasas esenciales para la nutrición humana. En la tabla 3, se puede observar que el contenido graso de los especímenes varía de $1.52 \%$ a $15.80 \%$, siendo la carachama la de menor contenido y maparate con palometa las de mayor contenido. En general, se pueden considerar como especies magras (menores del $2 \%$ a la carachama, corvina, paiche y arahuana, y semigrasas (menores del $5 \%$ ), a las especies lisa sábal o, sardina, yahuarachi, ractacara, dorado y yulilla; y especies grasas (mayores del 5\%), al paco, gamitana, palometa, boquichico y maparatc.

\subsubsection{Contenido de proteínas}

El componente más importante para la alimentación humana que contiene la carne de pescado, es su proteína, de gran valor biológico. El alto grado de aprovechamiento de éstas, obedece a la clase y relación existente entre los aminoácidos presentes en ella, sobre todo en lo referente a aminoácidos 

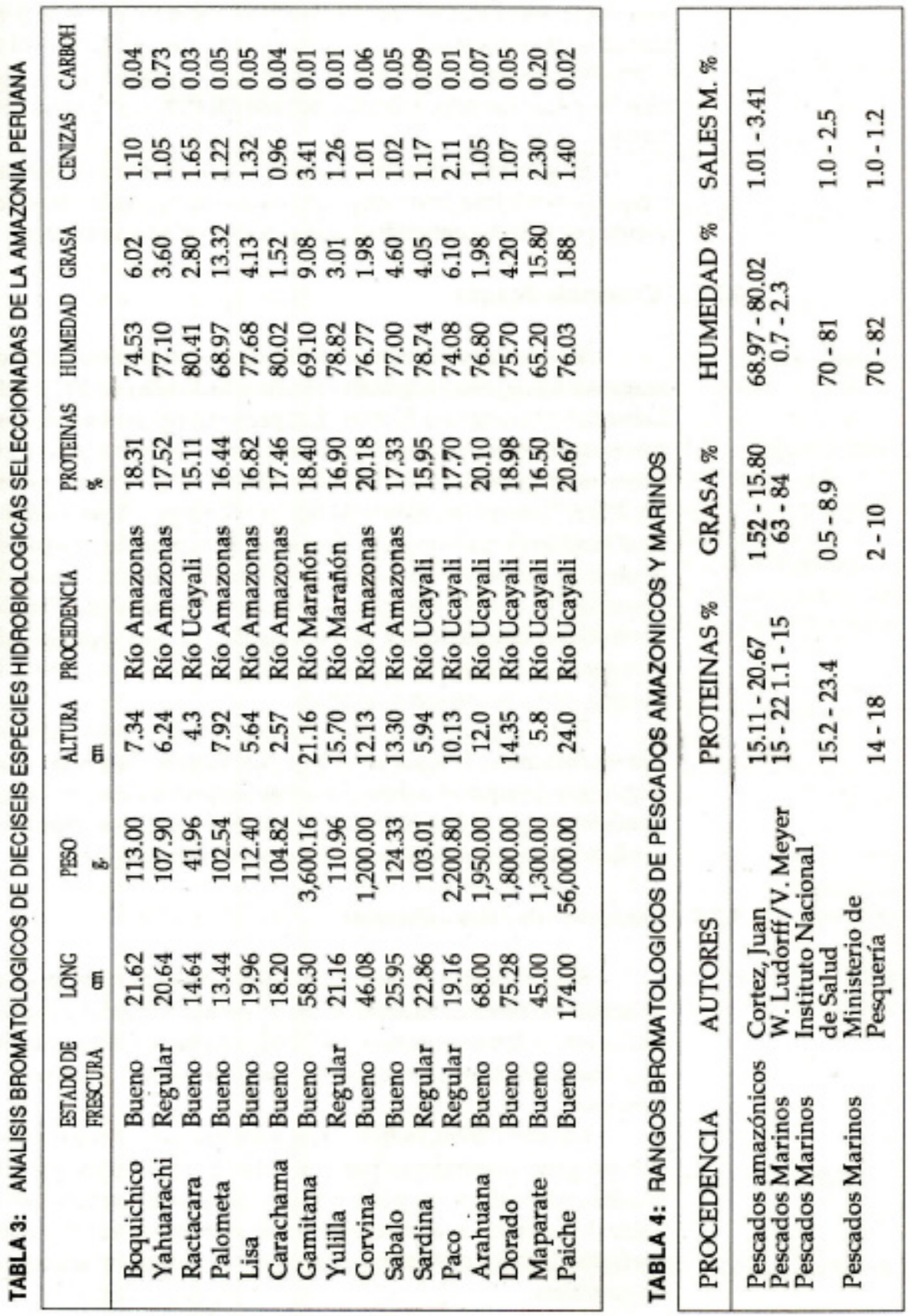
esenciales (W. Ludorff, M. M eyer 1978); según la Tabla 3 el contenido de ractacara el menor valor, con $15.61 \%$ y los mayores a la corvina, con $20.18 \%$ y al paiche con $20.61 \%$.

En general, la diferencia en porcentaje proteico de especie a especie es mínima, con excepción de estas dos especies, posiblemente por el bajo contenido graso y de humedad que poseen.

\subsubsection{Contenido de agua}

Se aprecia que el contenido de agua es mayor en los peces magros y semigrasos, llegando a alcanzar la carachama $80.02 \%$ de humedad y la ractacara $80.71 \%$. Los peces semigrasos mantienen cierto equilibrio de contenido de agua, pero es bastante baja en los peces muy grasos, como la palometa $(68.97 \%)$ y el maparate $(56.20 \%)$. Es importante lo señalado por L udore y M ayer, Ç 1978), en el sentido de que los peces aumentan su contenido de agua en el período de desove, a la vez que desciende el porcentaje de proteínas, ya que en esta epoca son extraordinariamente altas las necesidades energéticas y como además no hay ingestión de alimentos, el organismo se ve en la necesidad de recurrir a la poteína como sustancia energética.

En tal sentido, en la amazonía peruana, los peces disminuyen su contenido de agua en la fase de vaciante. Este aspecto es importante porque va a determinar variaciones en los otros componentes, según la fase hidrobiológica de captura. Asimismo, va a influir en los rendimientos en procesamiento pesquero.

\subsubsection{Contenido de sales minerales}

Según Vallejos y Menchola, (1984), Ias sustancias minerales actúan como catalizadores en los procesos biológicos y ejercen acción estimulante sobre la actividad de muchas enzimas; asimismo, intervienen en la regulación de la actividad muscular y nerviosa.

L udorff (1978), indica al respecto, que las sales minerales son de gran importancia para la vida de las células, para la construcción del cuerpo y la formación de huesos y sangre. En la Tabla 3 se aprecia que el contenido de sales minerales (cenizas) varía de $1.01 \%$ a 3.41\%, siendo menor en yahuarachí y el máximo en gamitana. 


\section{CONCLUSIONES Y RECOMENDACIONES}

Según el contenido graso en época de creciente, las especies amazónicas estudiadas quedan clasificadas en I ta forma siguiente:

- M agras (menores de 2\%): carachama, corvina, paiche, arahuana.

- Semigrasas (menores del 5\%): lisa, sábalo, sardina, yahuarachi, ractacara, yuylilla y dorado.

- Crasas (mayores del 5\%): paco, gamitana, palometa, boquichico y maparate.

Según el contenido de humedad, de acuerdo a lo señalado por L udorff y M eyer (a mayor grasa es menor la humedad), los resultados indican que las especies guardan una relación inversamente proporcional entre grasa y humedad; así, se observa en la Tabla 3, que la carachama posee $80.02 \%$ de humedad y solamente $1.52 \%$ de grasa, en el caso contrario, el maparate posee $15.80 \%$ de grasa y solamente $65.2 \%$ de humedad.

Según el contenido proteico, se puede señalar en general, que el aporte que da a la alimentación humana el consumo de los recursos pesqueros de la amazonía peruana en proteínas es bastante alto, siendo los porcentajes obtenidos del 15 al 22\%, debiéndose considerar, además, lo señalado por Gorgstrom (1965), que el alto contenido de aminoácidos esenciales presentes en el pescado hace posible la fácil asimilación proteica del pescado por el organismo humano.

El contenido de sales minerales presente en los peces amazónicos es de gran importancia (entre $1.01 \%$ y $3.41 \%$ ), en razón de que contienen cantidades considerables de calcio, magnesio y fósforo, elementos que son indispensables para la vida celular, el desarrollo del cuerpo y de la estructura ósea. A simismo, es importante resaltar que, en general, el contenido de sales minerales de los peces amazónicos es mayor que los marinos (ver Tabla 4).

El contenido proteico y de grasa de las especies amazónicas no presenta gran diferencia con las marinas, manteniéndose entre los rangos señalados por varios autores (Ludorff, M eyer, op. cit.), pero si es notorio su mayor contenido de humedad y de sales minerales.

Considerando que la composición química del pescado varía en relación a diferentes factores (edad del pez, régimen alimenticio, estaciones, etc.), se recomienda repetir estos análisis en diferentes épocas del año.

\section{BIBLIOGRAFIA}

BURGUES, G. 1965. El pescado y las industrias derivadas de la pesca. Ed. Acribia, Zaragosa, España. 280 pp.

LUDFORFF, W. 1978. El pescado y los productos de la pesca. Ed. Acribia, Zaragosa, España. 342 pp. 
MINISTERIO DE SALUD 1973. Institutos Nacionales de Salud, Instituto de Nutrición. $\mathrm{L}$ a composición de los alimentos peruanos 5 ta. Ed. Lima 35 pp.

INSTITUTO DEL MAR DEL PERU 1984. Composición y valor nutritivo de algunas especies pesqueras continentales. 6ta. Ed. Lima 8 pp.

CONNEL, J. 1972. Control de calidad del pescado. Ed. A cribia. Zaragosa, España. $270 \mathrm{pp}$.

INSTITUTO TECNOLOGICO PESQUERO DEL PERU 1988. Proteínas del músculo del pescado, 2da. Ed. Lima-Callao. $46 \mathrm{pp}$.

INSTITUTO TECNOLOGICO PESQUERO DEL PERU 1988. Composición química general del pescado. 3ra. Ed. Lima-Callao. $34 \mathrm{pp}$.

MINISTERIO DE PESQUERIA 1984. M anipuleo y preservación de pescado a bordo. 1ra. Ed. Lina $97 \mathrm{pp}$. 